\title{
Molecular Docking Studies of Phytoconstituents Identified in Crocus sativus, Curcuma longa, Cassia occidentalis and Moringa oleifera on Thymidylate Synthase - An Enzyme Target for Anti-Cancer Activity
}

\author{
Nikitha K. Heble, Rishitha Chowdary Mavillapalli, Ramesh Selvaraj, Srikanth Jeyabalan* \\ Department of Pharmacology, Faculty of Pharmacy, Sri Ramachandra University, Porur, Chennai - 600 116, Tamil Nadu, India.
}

\begin{tabular}{|c|c|}
\hline ARTICLE INFO & ABSTRACT \\
\hline $\begin{array}{l}\text { Article history: } \\
\text { Received on: } 25 / 06 / 2016 \\
\text { Revised on: } 08 / 11 / 2016 \\
\text { Accepted on: } 06 / 12 / 2016 \\
\text { Available online: } 28 / 12 / 2016 \\
\end{array}$ & $\begin{array}{l}\text { The aim and objective of the present study is to perform in-silico docking analysis of the major active } \\
\text { constituents identified in four Indian medicinal plants namely Crocus sativus, Curcuma longa, Cassia } \\
\text { occidentalis and Moringa oleifera for anti-cancer activity. In-silico docking analysis was performed by using } \\
\text { Molegro Virtual Docker (MVD). The parameter used for the docking analysis are MolDock score, Rerank score } \\
\text { and H-Bond interactions (binding energy). The target for anti-cancer activity is thymidylate synthase. The X- }\end{array}$ \\
\hline $\begin{array}{l}\text { Key words: } \\
\text { In-silico docking, } \\
\text { Phytoconstituents, Medicinal } \\
\text { Plants, Cancer, Molegro } \\
\text { Virtual Docker. }\end{array}$ & $\begin{array}{l}\text { Ray crystal co-ordinate of thymidylate synthase (PDB ID- } 1 \mathrm{HVY} \text { ) was retrieved from protein data bank in .pdb } \\
\text { format. The phytoconstituents of four medicinal plants were retrieved from PubChem compound database in } \\
\text {.mol format. The standard drugs Ralitrexed, 5-Fluorouracil and Vinblastine were obtained from the drug bank in } \\
\text {.mol format for comparison. The comparative anti-cancer activity of the phytoconstituents of four medicinal } \\
\text { plants are analysed by docking score and binding energy. It was analysed from the parameters of docking that } \\
\text { the phytoconstituents from Crocus sativum showed better anti-cancer activity compared to that of the standard } \\
\text { drugs. }\end{array}$ \\
\hline
\end{tabular}

\section{INTRODUCTION}

Cancer also known as a malignant tumor is a group of diseases involving abnormal cell growth with the potential to invade or spread to other parts of the body. Not all tumours are cancerous; benign tumors do not spread to other parts of the body. The number of new cancer cases ranges from 3.7 million in Eastern Asia to about 1800 in Micronesia/Polynesia.

Recently, The National Cancer Registry Programme (NCRP) commenced by the Indian Council of Medical Research, Bangalore, has published a report on Time Trends in Cancer Incidence Rates and it revealed that the total cancer cases are likely to go up from 979,786 cases in the year 2010 to $1,148,757$ cases in the year 2020 (Takiar et al., 2010). Thymidylate synthase is a key enzyme in the synthesis of 2'-deoxythymidine-

\footnotetext{
* Corresponding Author

E-mail:srikanthcologist@gmail.com
}

5'-monophosphate, an essential precursor for DNA biosynthesis. The improved knowledge of the complex mechanism of the biological pathways in which thymidylate synthase is involved represents a unique chance to find new mechanism-based inhibitors, aimed to treat cancer. For this reason, this enzyme is a critical target in cancer chemotherapy. The most widely used inhibitor of thymidylate synthase is 5-Fluorouracil (5-FU) (Kawakami et al., 2001). Recent years have witnessed a renewed interest in plants as pharmaceuticals in the Western world. Herbal medicines play a major role in primary health care, mainly in the developing countries. Therapeutic potential of herbal drugs are attributed to the bioactive phytochemical present in it. Plants are biosynthetic laboratories of a wide spectrum of chemicals of various physiological functions. These phytochemical are believed to have better compatibility with the human body and possess medicinal properties. Herbal drugs have a successful history as old as human civilization and today herbal medicines are coming back into prominence because of decreasing efficacy and serious side effects of the modern medicines (John et al., 2012). 
Saffron is a spice derived from the flower of the saffron crocus (Crocus sativus) plant native to Southwest Asia. Significant information points to the ability of saffron to inhibit cancer (Abdullaev, 2003). Aqueous saffron preparations have been reported to inhibit chemically induced skin carcinogenesis (Das et al., 2004). Both changes in carcinogen bio activation and tumour proliferation appear to occur (Das et al., 2010). Curcumin (diferuloylmethane) is a polyphenol derived from the Curcuma longa plant, commonly known as turmeric. Curcumin, widely used as a spice and colouring agent in food, possesses potent antioxidant, anti-inflammatory and anti-tumour promoting activities (Kuo et al., 1996). Cassia occidentalis L. is an annual or perennial ayurvedic plant which is used in several traditional medicines to cure various diseases. This weed has been known to possess antibacterial, antifungal, antidiabetic, anti-inflammatory, anticancerous, antimutagenic and hepatoprotective activity (Yadav et al., 2010). Moringa oleifera Lam (Moringaceae) is a highly valued plant, distributed in many countries of the tropics and subtropics. It has an impressive range of medicinal uses with high nutritional value. In addition to its compelling water purifying powers and high nutritional value, Moringa oleifera is very important for its medicinal value (Anwar et al., 2007).

The aim and objective of the present study is to perform in-silico docking analysis of the major active constituents identified in four Indian medicinal plants namely Crocus sativus, Curcuma longa, Cassia occidentalis and Moringa oleifera for anticancer activity on the enzyme target thymidylate synthase and compare the docking score and binding patterns with that of the standard drugs Ralitrexed, 5-Fluorouracil and Vinblastine.

\section{MATERIALS and METHODS}

\section{Molecular docking studies \\ Preparation of Ligand}

The major phytoconstituents are identified from the selected medicinal plants namely crocin, crocetin, picrocrocin from Crocus sativus (Escriban et al., 1996), curcumin, DL-arturmerone, isagarin from Curcuma longa (Nutakul, 2013), pentalogin, scopoletin from Cassia occidentalis (Dholwani et al., 2008) and niazimicin, niazirin from Moringa oleifera (Hussain et al., 2014) which possess anti cancer properties according to traditional claims. The 3D structures of the active constituents are retrieved from PubChem chemical databases (Bolton et al., 2008) and saved in .mol format. The ligands are imported to the workspace and preparation is done for docking studies. The docking scores of the active constituents are compared against the standard drugs (Ralitrexed, Vinblastine, 5- Fluorouracil) obtained from the drug bank in .mol format (Wishart et al., 2008).

\section{Preparation of Enzyme}

The target for docking studies is selected as thymidylate syntahse. Docking analysis is done by initially selecting the target for the disease and followed by obtaining the 3D structure of thymidylate syntahse (1HVY) (Phan et al., 2001) from protein data bank (Bernstein et al., 1978) in .pdb format. It is well known that PDB files often have poor or missing assignments of explicit hydrogens, and the PDB file format cannot accommodate bond order information.

Therefore, proper bonds, bond orders, hybridization and charges were assigned using the MVD. The potential binding sites of both the targets were calculated using the built-in cavity detection algorithm implemented in MVD. The search space of the simulation exploited in the docking studies was studied as a subset region of 25.0 Angstroms around the active side cleft. The water molecules are also taken in to consideration and the replaceable water molecules were given a score of 0.50 .

\section{Molegro Virtual Docker's docking search algorithms and scoring functions}

Ligand docking studies were performed by Molegro Virtual Docker (MVD), which has recently been introduced and gained attention among medicinal chemists. MVD is a fast and flexible docking program that gives the most likely conformation of ligand binding to a macromolecule. MolDock software is based on a new heuristic search algorithm that combines differential evolution with a cavity prediction algorithm (Thomsen and Christensen, 2006). It has an interactive optimization technique inspired by Darwinian Evolution Theory (Evolutionary Algorithms - EA), in which a population of individuals is exposed to competitive selection that weeds out poor solutions. Recombination and mutation are used to generate new solutions. The scoring function of MolDock is based on the Piecewise Linear Potential (PLP), which is a simplified potential whose parameters are fit to protein-ligand structures and a binding data scoring function (Gehlhaar et al., 1998) that is further extended in GEMDOCK (Generic Evolutionary Method for molecular DOCK) (Yang and Chen, 2004) with a new hydrogen bonding term and charge schemes.

\section{MolDock Optimizer}

In MVD, selected parameters were used for the guided differential evolution algorithm: number of runs $=5$ by checking constrain poses to cavity option), population size $=50$, maximum interactions $=2000$, cross over rate $=0.9$, and scaling factor $=0.5 . \mathrm{A}_{\mathrm{o}}$ variance-based termination scheme was selected rather than root mean square deviation (RMSD).To ensure the most suitable binding mode in the binding cavity, Pose clustering was employed, which lead to multiple binding modes.

\section{Parameters for scoring functions MolDock score}

They ignore-distant-atoms option was used to ignore atoms far away from the binding site. Additionally, hydrogen bond directionality was said to check whether hydrogen bonding between potential donors and acceptors can occur. The binding site on the protein was defined as extending in $\mathrm{X}, \mathrm{Y}$ and $\mathrm{Z}$ directions around the selected cavity with a radius of 25 Angstroms. 


\section{Rerank Score}

The reranking scoring functions are used to create and predict models for estimation of chemical properties (e.g. QSAR). The reranking score function is computationally more expensive than the scoring function used during the docking simulation but it is generally better than the docking score function at determining the best pose among several poses originating from the same ligand. While the rerank-score in MVD provides an estimate of the strength of the interaction, it is not calibrated in chemical units and it does not take complex contributions (such as entropy) into account. Even though the rerank score might be successful in ranking different poses of the same ligand, it might be less successful in ranking poses of different ligands. It is therefore recommend ranking the results of a virtual screening run using the rerank score. The binding affinity measure may then be used subsequently to get a rough estimate of the highest ranked poses.

\section{DruLiTo Software}

DruLiTo is open source software. It can calculate different molecular properties and screen the molecules based on drug likeness rules such as, 'The Lipinski rule of five' (Lipinski 2004), MDDR-like rule, Veber Rule, Ghose Filter, BBB Rule, CMC-50 like rule and Quantitative estimate of drug likeness (QED).

\section{RESULTS and DISCUSSIONS}

\section{In-silico docking results}

The ability of the phytoconstituents to bind with the targets is given in terms of MolDock Score. The MolDock Score and re rank scoring are used as the parameters for analysing the docking results. The phytoconstituents are ranked according to their MolDock Score. The ligand possessing the highest mol dock and re rank score shows a strong affinity towards its target.

In-silico docking analysis of phytoconstituents from Crocus sativus, Curcuma longa, Cassia occidentalis and Moringa oleifera on thymidylate syntahse (PDB ID: 1HVY) ranking based on MolDock Score is represented in table 1 and HBond is represented in table 2 . The binding pattern of crocetin are analysed using the ligand energy inspector tool built-in in the Molegro virtual docker. It was found that the binding patterns are similar to that of the standard drug Ralitrexed and it poses maximum MolDock Score as well as the rerank score. The structure 1HVY has in total 4 chains (A, B, C and D chains). Each chain of Thymidylate synthase possesses 288 residues. It was analysed that the chain $\mathrm{C}$ plays a major role in the binding to phytoconstituents including the standard drug.

The residues in the 1HVY chain $\mathrm{C}$ which are involved in the binding to the standard drug ralitrexed and crocetin are Ala 312, Arg 50, Arg 126, Arg 215, Asn 112, Asn 226, Asp 48, Asp 49, Asp 218, Asp 254, Cys 195, Gln 214, Glu 87, Glu 310, Gly 217, Gly 222, His 196, His 256, His 261, Ile 108, Leu 192, Leu 221, Lys 47, Lys 77, Lys 107, Lys 308, Met 311, Phe 80, Phe 225, Ser 216, Thr 51, Trp 109, Tyr 258, Arg 163, Arg 175, Arg 176, Asp 173, Asp174.The binding pattern for Ralitrexed, Crocetin, Curcumin and Niazimicin are represented in the figure 1,2,3 and 4 respectively.

The 'drug likeness properties' of the phytoconstituents was evaluated according to the 'The Lipinski rule of five' and to develop them as potential lead compound for anti-cancer activity. The rules, based on the 90-percentile values of the drug's property distributions, apply only to absorption by passive diffusion of compounds through cell membranes; compounds that are actively transported through cell membranes by transporter proteins are exceptions to the rule. The Lipinski criteria are widely used by medicinal chemists to predict not only the absorption of compounds, as Lipinski originally intended, but also overall druglikeness. The results of the phytoconstituents analysed are represented in table 3 .

All the phytoconstituents passes the drug likeness properties except Vinblastine and crocin.

Further studies can be performed to evaluate the in-vitro and in-vivo anticancer activity of the selected medicinal plants and to discover pharmacokinetic properties of the phytoconstituents to know the absorption, distribution, metabolism and excretion of the phytoconstituents.

Table 1: In-silico docking analysis of phytoconstituents from Crocus sativus, Curcuma longa, Cassia occidentalis and Moringa oleifera thymidylate synthase (PDB ID: 1HVY) ranking based on MolDock Score.

\begin{tabular}{|c|c|c|c|c|c|}
\hline S. No. & Name & Ligand & MolDock Score & Rerank Score & HBond \\
\hline 1. & [01]Ralitrexed & Ralitrexed & -173.887 & -128.979 & -11.2637 \\
\hline 2. & {$[00]$ Crocetin } & Crocetin & -125.41 & -105.362 & -5.46598 \\
\hline 3. & [01]Curcumin & Curcumin & -106.289 & -82.4104 & -3.746 \\
\hline 4. & [00]Niazimicin & Niazimicin & -104.745 & -94.2493 & -6.91162 \\
\hline 5. & [00]Picrocrocin & Picrocrocin & -101.803 & -88.7085 & -6.65758 \\
\hline 6. & [00]Vinblastine & Vinblastine & -91.9291 & 56.8852 & -3.59816 \\
\hline 7. & [00]Niazirin & Niazirin & -89.8043 & -88.3942 & -6.41756 \\
\hline 8. & [00]Turmerone & Turmerone & -89.0163 & -73.2264 & -0.219559 \\
\hline 9. & [00]Scopoleptin & Scopoleptin & -77.1298 & -66.3236 & -4.2016 \\
\hline 10. & {$[00]$ crocin } & crocin & -65.728 & -25.4591 & -10.499 \\
\hline 11. & [00]Fluorouracil & Fluorouracil & -55.7535 & -50.5361 & -2.08843 \\
\hline 12. & [00]Pentalogin & Pentalogin & 1971.95 & 44.9628 & -0.442231 \\
\hline
\end{tabular}


Table 2: In-silico docking analysis of phytoconstituents from Crocus sativus, Curcuma longa, Cassia occidentalis and Moringa oleifera thymidylate synthase (PDB ID: 1HVY) ranking based on HBond interaction.

\begin{tabular}{|c|c|c|c|c|c|}
\hline S. No. & Name & Ligand & MolDock Score & Rerank Score & HBond \\
\hline 1. & [01]Ralitrexed & Ralitrexed & -173.887 & -128.979 & -11.2637 \\
\hline 2. & {$[00]$ crocin } & crocin & -65.728 & -25.4591 & -10.499 \\
\hline 3. & [00]Niazimicin & Niazimicin & -104.745 & -94.2493 & -6.91162 \\
\hline 4. & [00]Picrocrocin & Picrocrocin & -101.803 & -88.7085 & -6.65758 \\
\hline 5. & [00]Niazirin & Niazirin & -89.8043 & -88.3942 & -6.41756 \\
\hline 6. & [00]Crocetin & Crocetin & -125.41 & -105.362 & -5.46598 \\
\hline 7. & [00]Scopoleptin & Scopoleptin & -77.1298 & -66.3236 & -4.2016 \\
\hline 8. & [01]Curcumin & Curcumin & -106.289 & -82.4104 & -3.746 \\
\hline 9. & [00]Vinblastine & Vinblastine & -91.9291 & 56.8852 & -3.59816 \\
\hline 10. & [00]Fluorouracil & Fluorouracil & -55.7535 & -50.5361 & -2.08843 \\
\hline 11. & [00]Pentalogin & Pentalogin & 1971.95 & 44.9628 & -0.442231 \\
\hline 12. & [00]Turmerone & Turmerone & -89.0163 & -73.2264 & -0.219559 \\
\hline
\end{tabular}

Table 3: The Lipinski rule of five for evaluation of 'drug likeness properties' using DruLiTo Software.

\begin{tabular}{|c|c|c|c|c|c|}
\hline Sr. No. & Title & MW & $\log P$ & HBA & HBD \\
\hline 1 & Crocetin & 328.17 & 4.472 & 4 & 2 \\
\hline 2 & Curcumin & 368.13 & 1.945 & 6 & 2 \\
\hline 3 & Turmerone & 216.15 & 3.669 & 1 & 0 \\
\hline 4 & Niazimicin & 357.12 & 0.592 & 7 & 4 \\
\hline 5 & Pentalogin & 285.21 & 2.723 & 2 & 1 \\
\hline 6 & Picrocrocin & 330.17 & -0.207 & 7 & 4 \\
\hline 7 & Scopoleptin & 192.04 & 0.97 & 4 & 1 \\
\hline 8 & Fluorouracil & 130.02 & -1.137 & 4 & 2 \\
\hline 9 & Niazirin & 269.13 & 0 & 6 & 4 \\
\hline 10 & Vinblastine & 753.98 & 3.056 & 13 & $\mathbf{0}$ \\
\hline 11 & Ralitrexed & 452.08 & -1.314 & 4 & 3 \\
\hline 12 & Crocin & 976.38 & -1.214 & 24 & 14 \\
\hline
\end{tabular}

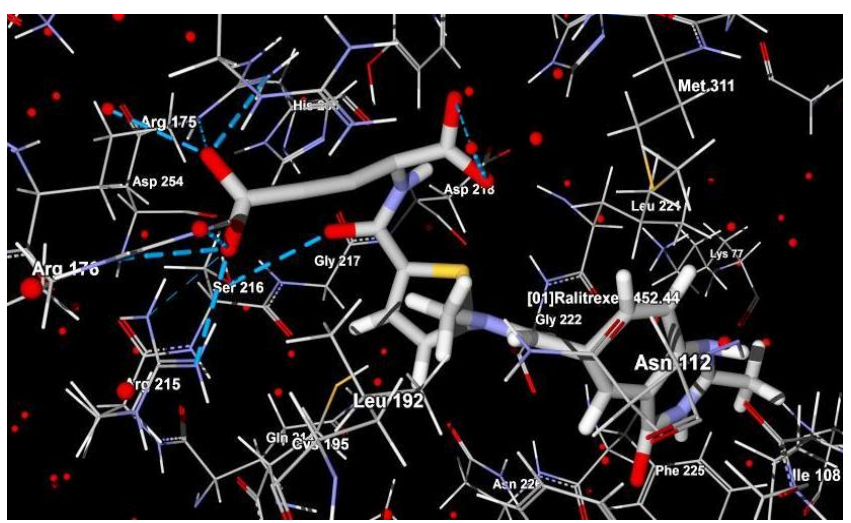

Fig. 1: Docked view of Ralitrexed with the enzyme Thymidylate synthase $(1 \mathrm{HVY})$

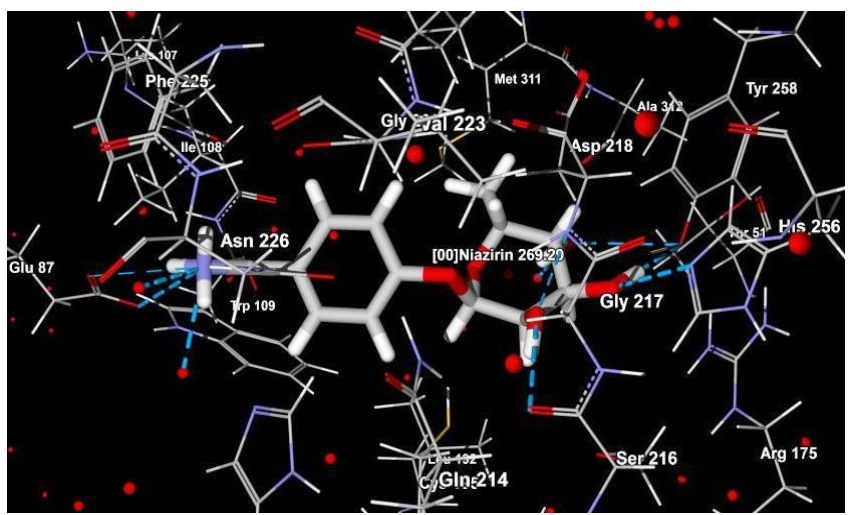

Fig. 3: Docked view of Curcumin with the enzyme Thymidylate synthase (1HVY)

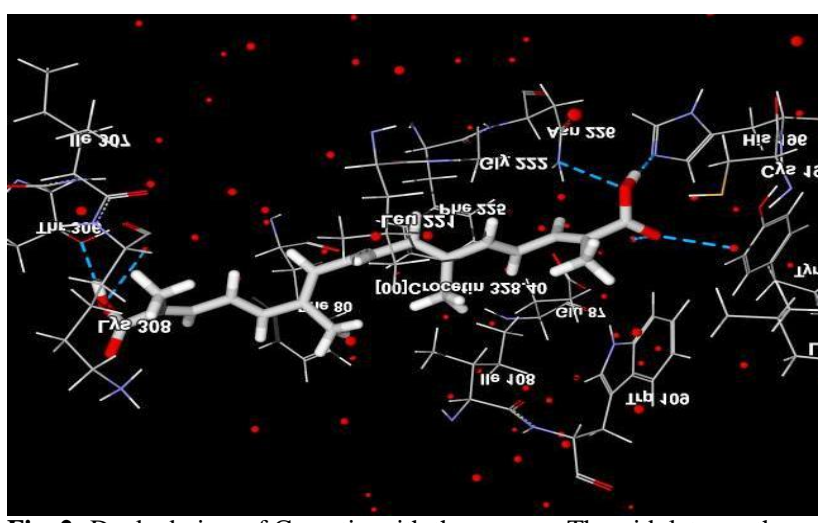

Fig. 2: Docked view of Crocetin with the enzyme Thymidylate synthase (1HVY)

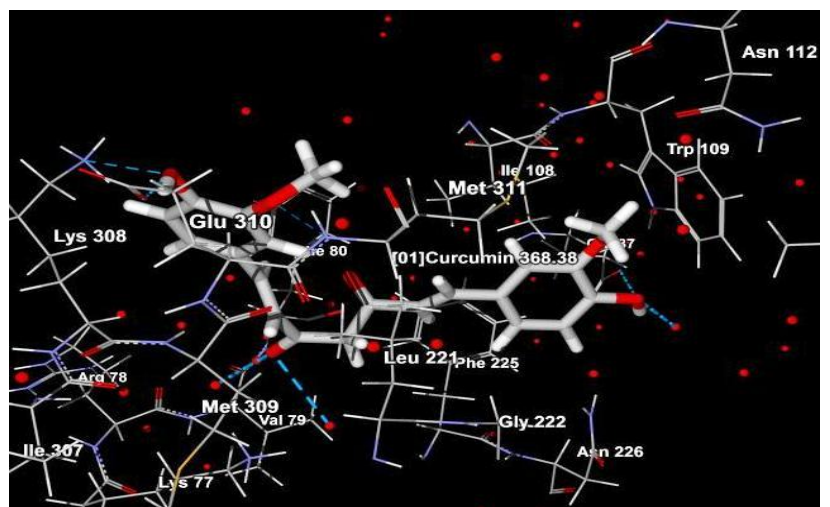

Fig. 4: Docked view of Niazirin with the enzyme Thymidylate synthase (1HVY) 


\section{ACKNOWLEDGEMENTS}

The authors thank the management of Sri Ramachandra University for providing us with all the facilities for the successful completion of the project.

\section{Financial support and sponsorship: Nil}

Conflict of Interests: There are no conflicts of interest.

\section{REFERENCES}

Abdullaev F. Crocus sativus against cancer. Archives of medical research, 2003; 34(4):354.

Anwar F, Latif S, Ashraf M, and Gilani AH. Moringa oleifera: a food plant with multiple medicinal uses. Phytotherapy research, 2007; 21(1): $17-25$

Bernstein FC, Koetzle TF, Williams GJ, Meyer EF, Brice MD, Rodgers JR and Tasumi M. The Protein Data Bank: a computer-based archival file for macromolecular structures. Archives of biochemistry and biophysics, 1978; 185(2): 584-591.

Bolton EE, Wang Y, Thiessen PA and Bryant SH. PubChem: integrated platform of small molecules and biological activities. Annual reports in computational chemistry, 2008; 4: 217-241.

Das I, Chakrabarty RN, and Das S. Saffron can prevent chemically induced skin carcinogenesis in Swiss albino mice. Asian Pac J Cancer Prev, 2004; 5(1): 70-76.

Das I, Das S, and Saha T. Saffron suppresses oxidative stress in DMBA-induced skin carcinoma: a histopathological study. Acta histochemica, 2010; 112(4): 317-327.

Dholwani, K. K., Saluja, A. K., Gupta, A. R., and Shah, D. R. A review on plant-derived natural products and their analogs with anti-tumor activity. Indian journal of pharmacology, 2008; 40(2): 49.

Escribano, J., Alonso, G. L., Coca-Prados, M., and Fernández, J. A.. Crocin, safranal and picrocrocin from saffron (Crocus sativus L.) inhibit the growth of human cancer cells in vitro. Cancer letters, 1996; 100(1): 23-30.

Gehlhaar DK, Bouzida D, and Rejto PA. Fully automated and rapid flexible docking of inhibitors covalently bound to serine proteases. In Evolutionary Programming VII, 1998, January; (pp. 449-461). Springer Berlin Heidelberg.

Hussain, S., Malik, F., and Mahmood, S. Review: an exposition of medicinal preponderance of Moringa oleifera (Lank.). Pakistan journal of pharmaceutical sciences, 2014; 27(2): 397-403.

John J, Mehta A, and Mehta P. Evaluation of antioxidant and anticancer potential of Cassia tora leaves. Asian Journal of Traditional Medicines, 2012; 7(6): 260-267.
Kuo ML, Huang TS, and Lin JK. Curcumin, an antioxidant and anti-tumor promoter, induces apoptosis in human leukemia cells. Biochimica et Biophysica Acta (BBA)-Molecular Basis of Disease, 1996; 1317(2): 95-100.

Kawakami K, Salonga D, Park JM, Danenberg KD, Uetake H, Brabender J and Danenberg PV. Different lengths of a polymorphic repeat sequence in the thymidylate synthase gene affect translational efficiency but not its gene expression. Clinical cancer research, 2001; 7(12): 40964101.

Lipinski CA. Lead-and drug-like compounds: the rule-of-five revolution. Drug Discovery Today: Technologies, 2004; 1(4): 337-341.

Nutakul, W. NMR Analysis of Antipeptic Ulcer Principle from Curcuma longa L. Bulletin of the Department of Medical Sciences, 2013; 36(4): 211-2

Phan J, Koli S, Minor W, Dunlap RB, Berger SH and Lebioda L. Human thymidylate synthase is in the closed conformation when complexed with dUMP and raltitrexed, an antifolate drug. Biochemistry, 2001; 40(7): 1897-1902.

Thomsen R, and Christensen MH. MolDock: a new technique for high-accuracy molecular docking. Journal of medicinal chemistry, 2006; 49(11): 3315-3321.

Takiar R, Nadayil D and Nandakumar A. Projections of number of cancer cases in India (2010-2020) by cancer groups. Asian Pac J Cancer Prev, 2010; 11(4):1045-1049.

Wishart DS, Knox C, Guo AC, Cheng D, Shrivastava S, Tzur D and Hassanali M. DrugBank: a knowledgebase for drugs, drug actions and drug targets. Nucleic acids research, 2008; 36(suppl 1): D901-D906.

Yang JM, and Chen CC. GEMDOCK: a generic evolutionary method for molecular docking. Proteins: Structure, Function, and Bioinformatics, 2004; 55(2): 288-304.

Yadav JP, Arya V, Yadav S, Panghal M, Kumar S and Dhankhar S. Cassia occidentalis L.: A review on its ethnobotany, phytochemical and pharmacological profile. Fitoterapia, 2010; 81(4): 223 230 .

\section{How to cite this article:}

Heble NK, Mavillapalli RC, Selvaraj R, Jeyabalan S. Molecular Docking Studies of Phytoconstituents Identified in Crocus sativus, Curcuma longa, Cassia occidentalis and Moringa oleifera on Thymidylate Synthase: An Enzyme Target for Anti-Cancer Activity. J App Pharm Sci, 2016; 6 (12): 131-135. 